\title{
Multicell OFDMA Downlink Resource Allocation Using a Graphic Framework
}

\author{
Ronald Y. Chang, Zhifeng Tao, Member, IEEE, Jinyun Zhang, Fellow, IEEE, and C.-C. Jay Kuo, Fellow, IEEE
}

\begin{abstract}
A novel practical low-complexity multicell orthogonal frequency-division multiple access (OFDMA) downlink channel-assignment method that uses a graphic framework is proposed in this paper. Our solution consists of two phases: 1) a coarse-scale intercell interference (ICI) management scheme and 2) a fine-scale channel-aware resource-allocation scheme. In the first phase, state-of-the-art ICI management techniques such as ICI coordination (ICIC) and base-station cooperation (BSC) are incorporated in our framework. In particular, the ICI information is acquired through inference from the diversity set of mobile stations and is presented by an interference graph. Then, ICIC or BSC is mapped to the MAX $k$-CUT problem in graph theory and is solved in the first phase. In the second phase, channel assignment is accomplished by taking instantaneous channel conditions into account. Heuristic algorithms are proposed to efficiently solve both phases of the problem. Extensive simulation is conducted for various practical scenarios to demonstrate the superior performance of the proposed solution compared with the conventional OFDMA allocation scheme. The proposed scheme can be used in next-generation cellular systems such as the 3GPP Long-Term Evolution and IEEE 802.16m.
\end{abstract}

Index Terms-Base-station cooperation (BSC), cellular networks, graph theory, IEEE 802.16, intercell interference coordination (ICIC), interference management, orthogonal frequency-division multiple access (OFDMA), resource allocation.

\section{INTRODUCTION}

$\mathbf{O}$ RTHOGONAL frequency-division multiple access (OFDMA) is a widely adopted technology in many next-generation cellular systems such as the 3GPP LongTerm Evolution (LTE) [1] and IEEE 802.16m [2] due to its effectiveness and flexibility in radio resource allocation, as well as its capability to combat frequency-selective fading. The radio spectrum is a scarce resource in wireless communications, and therefore, its efficient use is critical. The rapid growth of wireless applications and subscribers has called for a good radio resource management (RRM) scheme that can increase the network capacity and, from a commercial point of

Manuscript received May 14, 2008; revised November 8, 2008. First published February 2, 2009; current version published August 14, 2009. This work was presented in part at the 2008 IEEE Global Telecommunications Conference, New Orleans, LA, November 30-December 4, 2008. The review of this paper was coordinated by Prof. V. W. S. Wong.

R. Y. Chang and C.-C. J. Kuo are with the Ming Hsieh Department of Electrical Engineering and the Signal and Image Processing Institute, University of Southern California, Los Angeles, CA 90089-2564 USA (e-mail: yjrchang@gmail.com; cckuo@sipi.usc.edu).

Z. Tao and J. Zhang are with the Digital Communications and Networking Group, Mitsubishi Electric Research Laboratories, Cambridge, MA 02139 USA (e-mail: tao@merl.com; jzhang@merl.com).

Color versions of one or more of the figures in this paper are available online at http://ieeexplore.iee.org.

Digital Object Identifier 10.1109/TVT.2009.2014384 view, save the deployment cost. Consequently, developing an effective radio resource-allocation scheme for OFDMA is of significant interest to academia and industry.

The fundamental challenge of resource allocation lies in the scarcity of the available spectrum, the expansive servicing area, and the large user number. As a result, the same frequency spectrum needs to often be reused in multiple geographical areas or cells. This will incur intercell interference (ICI) when users or mobile stations (MSs) in adjacent cells use the same spectrum. In fact, ICI has been shown to be the predominant performance-limiting factor in wireless cellular networks [3]. A significant amount of research has been devoted to ICI-aware radio resource allocation in cellular networks [3].

Although the techniques surveyed in [3] are useful in some application scenarios, many methods (e.g., channel borrowing) cannot directly be applied to networks using a frequency reuse-1 in cell deployment (i.e., the same spectrum is reused in each and every cell). Reuse- $n(n>1)$ systems tend to lose more spectral efficiency by segregating bandwidth than to gain better link quality by reducing interference; thus, reuse-1 has, in general, been considered as the preferred scheme for modern cellular OFDMA systems. Nevertheless, users of reuse-1 OFDMA networks, particularly those in the cell edge, admittedly suffer more severe ICI compared with users of reuse- $n(n>1)$ systems. Thus, a good ICI management scheme on top of OFDMA is needed.

Research endeavors on multicell OFDMA resource allocation with ICI consideration can be classified into two categories. The first category extends the single-cell allocation experience [4]-[6] to the multicell scenario, mainly by considering the signal-to-interference-plus-noise ratio (SINR) instead of the signal-to-noise ratio (SNR). This formulation is handy, because most of the single-cell OFDMA resource-allocation schemes can directly be applied to the multicell context. For instance, $\mathrm{Li}$ and $\mathrm{Liu}$ [7] proposed a two-level resource-allocation scheme where the radio network controller (RNC) coordinates multiple cells in the first level and performs per-cell optimization in the second level. The first level is conducted based on perfect and predetermined knowledge of the SINR for all MSs on all subchannels. In [8], a similar approach was adopted, with some special treatment on ICI. Pietrzyk and Janssen [9], [10] proposed heuristic algorithms for their formulated problems based on SINR, with some quality-of-service (QoS) consideration. Abrardo et al. [11] proposed a centralized and a distributed method for multicell OFDMA resource allocation based on the measurement of ICI. Note that one key assumption in this category of research is the availability of SINR. This assumption may be difficult to obtain a priori, however, because 


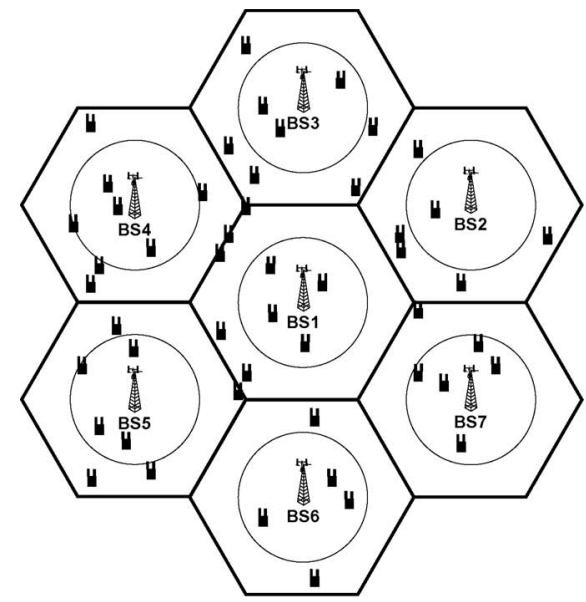

(a)

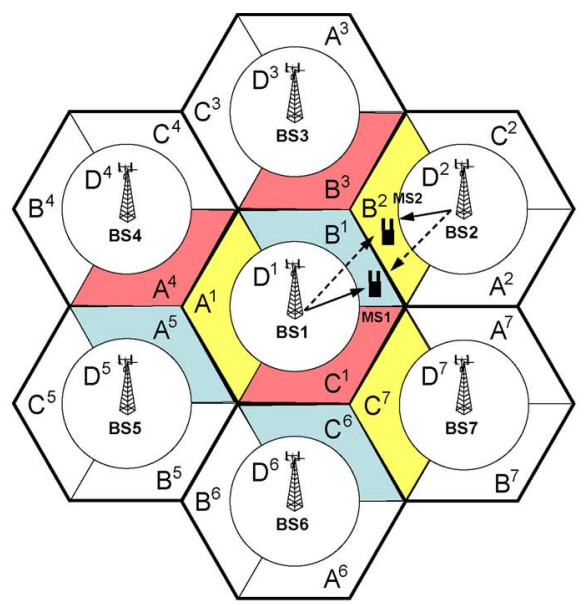

(b)

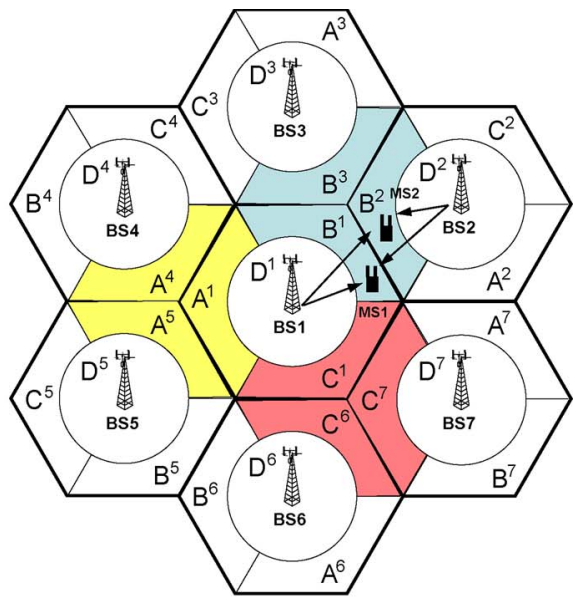

(c)

Fig. 1. Hexagonal multicell OFDMA cellular network and the associated resource management therein. (a) Multicell OFDMA cellular network. (b) ICIC. (c) BSC.

the interference depends on the distance, location, and occupied channel status of interferers, which are unknown before resource allocation. In other words, it is the mutual dependency of ICI that complicates the problem. Thus, a multicell resource allocation scheme that is contingent upon global and perfect knowledge of SINR may not be practical.

The second category of work aims at developing systematic RRM techniques and policies as guidelines for resource allocation. For instance, advanced techniques such as ICI coordination (ICIC) [12] and base-station cooperation (BSC) [13] were proposed to mitigate formidable ICI and improve the overall system performance. Similar RRM mechanisms were suggested for the multicell scenario in the 3GPP [14], [15]. Recently, new improvements have also been proposed in 3GPP LTE (e.g., [16] and [17]) and IEEE 802.16m (e.g., [18]) standardization activities. Some of the ICIC schemes (e.g., [17]) were designed based on the concept of soft reuse, i.e., asymmetric reuse factors are applied to cell-center and cell-edge regions. In particular, cell center is allowed to use a smaller reuse factor to enhance the spectral efficiency, because cell-center MSs, with reduced transceiving power, will cause less interference to neighbors, i.e., downlink power control (PC). The issues of downlink PC and soft reuse are further studied in [19] and [20]. Most research work in this category has concentrated on presenting the design concept of ICIC and/or BSC, justifying the use of these techniques, and obtaining the achievable performance bound. The problem of designing a practical algorithm that actually achieves the resource-allocation principle suggested by ICIC or BSC has largely been overlooked.

Based on this observation, we are motivated to propose a novel high-performance yet low-complexity multicell OFDMA downlink channel assignment method to enable ICIC and BSC in the reuse-1 deployment. In the proposed framework [21], the problem of ICI reduction is first addressed using a graphic approach, where no precise SINR information is required. Then, the task of channel assignment is conducted by taking instantaneous channel conditions into account. To strike a balance between performance optimality and practicality, heuristic algorithms are further proposed to simplify the solution of
ICI reduction and channel assignment. Extensive simulation demonstrates that the proposed scheme can offer substantial SINR improvements in the reuse-1 deployment.

The rest of this paper is organized as follows. After a brief background review in Section II, we describe our system model and the resource-allocation problem in Section III. Our solution framework is presented in Section IV. Two heuristic algorithms for facilitating the solution framework are discussed in Section V. The performance advantage of our proposed solution is demonstrated by computer simulation in Section VI. Finally, concluding remarks are given in Section VII.

\section{RESEARCH BACKGROUND}

\section{A. Multicell OFDMA Networks}

A hexagonal multicell OFDMA cellular network is considered in this paper. One example network with seven cells is drawn in Fig. 1(a). Each cell is served by a base station (BS) at the center of the cell, and there are a set of MSs within each cell. Based on its physical proximity to the BS, each MS is classified as either in the cell-center or the cell-edge area. The boundary that separates the cell center and the cell edge can be a design parameter. In OFDMA systems, the radio resource that will be allocated to users is the subchannel. A subchannel is a group of subcarriers that may or may not be contiguous, depending on the specific permutation scheme used, which determines the mapping from physical subcarriers to logical subchannels. As specified in the IEEE 802.16e standard [22], partial usage of subchannels (PUSC) and adaptive modulation and coding (AMC) are permutation schemes that define nonadjacent and adjacent subcarrier groupings for a subchannel, respectively.

\section{B. Diversity Set}

In regular operations, each MS is registered at and communicates with a single BS, which is called the anchor (or serving) BS. However, in some scenarios (e.g., soft handover or, as we will introduce later in this section, BSC), simultaneous communication with more than one BS may take 
place. A diversity set is defined in the 802.16e standard [22] to serve this purpose. It keeps track of the anchor BS and neighboring BSs that are within the communication range of an MS. This information is maintained at the MS and the BS. The diversity set of MS $m$ is denoted by $\mathbb{D}_{m}=\mathbb{A}_{m} \cup \mathbb{B}_{m}$, where $\mathbb{A}_{m}$ is the anchor BS set that has only one element (i.e., anchor BS $A_{m}$ ), and $\mathbb{B}_{m}$ is the neighbor BS set that may have zero, one, or multiple BSs. Note that the number of elements in set $\mathbb{B}_{m}$ depends on the geographic location of MS $m$ in relation to its neighboring BSs and on some path-loss threshold.

Property (Forming the Diversity Set): It is assumed that each MS, aside from its own serving BS, has at most two neighbor BSs in its diversity set. That is, for any MS $m,\left|\mathbb{D}_{m}\right| \leq 3$, where $|\cdot|$ is the cardinality of a set.

The aforementioned property follows from the observation about a hexagonal network that the dominant signal comes from the three nearest BSs. Signal from farther BSs will undergo more severe path-loss degradation before it reaches the MS and, thus, is assumed below the path-loss threshold used to determine the diversity set. Note, however, that when defining the SINR, we consider all BSs in the network as the potential interfering source. This consideration will be presented in Section III-A.

\section{C. $I C I C$}

ICI dominates the performance of interference-limited cellular networks; thus, proper ICI management is needed. ICIC was proposed in [12] and [16] to effectively reduce ICI in cell-edge regions. It is achieved by allocating disjoint channel resources to cell-edge MSs that belong to different cells. Celledge MSs are most prone to high ICI; thus, the overall ICI can be reduced by judicious coordination between cell-edge MSs in channel allocation. This idea is illustrated in Fig. 1(b), where same/different colors represent the use of the same/different subchannels of the band. MS 1 has anchor BS 1, and MS 2 has anchor BS 2. $A^{i}, B^{i}$, and $C^{i}$ refer to the three sectors ${ }^{1}$ in the cell-edge area, and $D^{i}$ refers to the cell-center area, with $i=$ $1, \ldots, 7$. Nonoverlapping channel resources, as shown by different colors, are allocated to MS 1 and MS 2 in neighboring $B^{1}$ and $B^{2}$ sectors, respectively. Therefore, the potential interference caused by downlink signals to each other, which is shown by dotted lines, is avoided. In general, ICIC suggests the allocation of disjoint channel resources to neighboring cell-edge regions (i.e., $A^{1}, A^{4}$, and $A^{5} ; B^{1}, B^{2}$, and $B^{3}$; and $C^{1}, C^{6}$, and $C^{7}$ ) to mitigate ICI. In other words, ICIC reduces the number of interferers and/or the "damage" of each interferer, which can be achieved by, for instance, allocating the same resource to MSs that are geographically farther from each other such that the interference is mitigated due to the increased path loss.

However, although ICIC that is solely based on cell-edge resource collision avoidance is beneficial to the uplink, it offers only a limited performance gain in the downlink scenario,

\footnotetext{
${ }^{1}$ The sectorization shown in Fig. 1(b) and (c) serves only to illustrate the relationship between the geographical location of MSs and resource management. We will focus on the discussion of nonsectorized cell deployment in the rest of this paper. Nevertheless, the framework established hereafter can readily be applied to sectorized systems.
}

because it overlooks the interference caused by transmission from the BS to cell-center MSs [16]. This tradeoff motivates us to develop a holistic channel assignment framework where all MSs, cell center and cell edge alike, are taken into account in ICIC management.

\section{D. $B S C$}

As proposed in [13] and [23], BSC is another effective ICI management scheme. BSC allows a group of BSs to concurrently send signals to a group of MSs, with each having an anchor BS from this group of BSs, using the same time and frequency resource. It can essentially be considered as a combination of space-division multiple access [24], [25] and macrodiversity handover [22], where multiple BSs simultaneously communicate to MSs that specifically reside in the celledge area and within the transmission range of the cooperating BSs. The concept of BSC is illustrated in Fig. 1(c). MS 1 has anchor BS 1, and MS 2 has anchor BS 2. The same channel resource (e.g., subchannels) represented by the same color is allocated to both MS 1 and MS 2 in cell-edge areas $B^{1}$ and $B^{2}$ of the neighboring BSs BS 1 and BS 2. Then, BS 1 and BS 2 jointly transmit signals to MS 1 and MS 2 in the same frequency band. Thus, the potential interference that would otherwise be caused by downlink signals to each other is now turned into useful signals, as shown by the solid lines in Fig. 1(c). Aside from ICI reduction, this method also achieves spatial diversity. In general, BSC suggests the allocation of overlapping channel resources to neighboring cell-edge regions (i.e., $A^{1}, A^{4}$, and $A^{5} ; B^{1}, B^{2}$, and $B^{3}$; and $C^{1}, C^{6}$, and $C^{7}$ ) to allow cooperation.

\section{System Model AND PROBlem DescRiPTION}

\section{A. System Model and SINR Derivation}

We consider a downlink hexagonal cellular network as described in Section II-A with $L$ BSs, each with $N_{T}$ antennas, and $M_{l}$ MSs, each with $N_{R}$ antennas, served by the $l$ th BS. The total number of MSs in the entire network is, therefore, $M=\sum_{l=1}^{L} M_{l}$. Each MS is labeled as either a cell-center or a cell-edge user, depending on its proximity to the BS. Assume that a set of $N$ subchannels is available for resource allocation, and the frequency reuse factor is one, i.e., each BS will use all $N$ subchannels. Note that due to the intracell allocation constraint in OFDMA networks, which restricts the use of a subchannel by at most one MS within the same cell, the number of served MSs in cell $l$ must be less than or equal to the number of subchannels, i.e., $M_{l} \leq N$ for all $l$ s.

Signal transmission in the multicell OFDMA system is modeled as follows. We consider an arbitrary symbol in an OFDMA frame for the interference study in the ensuing discussion. Let the $N_{R} \times N_{T}$ matrix $\mathbf{H}_{m n}^{(l)}$ represent the channel from BS $l$ to MS $m$ in the subchannel $n$, which has complex Gaussian elements. Let the $L_{m n} \times 1$ vector $\mathbf{s}_{m n}$ be the transmitted data intended for MS $m$ using subchannel $n$, which has zero mean and normalized power, i.e., $E\left[\mathbf{s}_{m n} \mathbf{s}_{m n}^{H}\right]=\mathbf{I}_{L_{m n}}$. The data vector $\mathbf{s}_{m n}$ is precoded by an $N_{T} \times L_{m n}$ precoding matrix $\mathbf{T}_{m n}$, which also has normalized power, i.e., $\left\|\mathbf{T}_{m n}\right\|_{F}^{2}=1$, where $\|\cdot\|_{F}^{2}$ is the Frobenius norm of a matrix. 
Suppose that downlink PC is employed to reduce the ICI caused to neighbor cells. That is, the downlink signal for MS $m$ is sent with power $P_{m}$, depending on its proximity to the BS. In particular, we have

$$
P_{m}= \begin{cases}P_{0}, & \text { if MS } m \text { is in the cell center } \\ P_{1}, & \text { if MS } m \text { is in the cell edge }\end{cases}
$$

where $P_{0}<P_{1}$.

In the ICIC operation, each MS communicates with one BS. Thus, the received baseband discrete-time signal at MS $m$ that uses subchannel $n$ after matched filtering and sampling comprises a useful signal part from the serving BS $A_{m}$ and the interference from the corresponding serving BS $A_{v}$ of the interfering MS $v$ plus noise, i.e.,

$$
\begin{aligned}
\mathbf{r}_{m n}=\sqrt{P_{m}} \mathbf{H}_{m n}^{\left(A_{m}\right)} & \mathbf{T}_{m n} \mathbf{s}_{m n} \\
& +\sum_{v \in \mathbb{I}_{m}} \sqrt{P_{v}} \mathbf{H}_{m n}^{\left(A_{v}\right)} \mathbf{T}_{v n} \mathbf{s}_{v n}+\mathbf{n}_{m n}
\end{aligned}
$$

where $\mathbb{I}_{m}$ is the set of interfering MSs for MS $m$, and $\mathbf{n}_{m n}$ is the additive white Gaussian noise with noise power $E\left[\mathbf{n}_{m n}^{H} \mathbf{n}_{m n}\right]=N_{0}$.

SINR is used to evaluate the performance of a multicell wireless cellular network. It is a more accurate measure compared with SNR in interference-limited cellular networks. Based on (2), the SINR (in the linear scale) of the received signal at MS $m$ that uses subchannel $n$ is given by

$$
\operatorname{SINR}_{m n}=\frac{P_{m}\left\|\mathbf{H}_{m n}^{\left(A_{m}\right)} \mathbf{T}_{m n}\right\|_{F}^{2}}{\sum_{v \in \mathbb{I}_{m}} P_{v}\left\|\mathbf{H}_{m n}^{\left(A_{v}\right)} \mathbf{T}_{v n}\right\|_{F}^{2}+N_{0}} .
$$

$\mathbf{H}_{m n}^{(l)}$ captures both slow-fading (due to path loss) and fastfading (due to the Rayleigh fading) effects; thus, it is convenient to consider the following equivalent form:

$$
\operatorname{SINR}_{m n}(\mathrm{ICIC})=\frac{P_{m} \beta_{m n}^{\left(A_{m}\right)} \varphi_{m}^{\left(A_{m}\right)}}{\sum_{v \in \mathbb{I}_{m}} P_{v} \beta_{m n}^{\left(A_{v}\right)} \varphi_{m}^{\left(A_{v}\right)}+N_{0}}
$$

where $\beta_{m n}^{(l)}$ is the fading channel power in subchannel $n$ from BS $l$ to MS $m$, and $\varphi_{m}^{(l)}$ is the path-loss attenuation factor from BS $l$ to MS $m$, independent of $n$. Note that ICIC aims at reducing the size of $\mathbb{I}_{m}$ (i.e., the number of interferers) and/or the "damage" of each interferer, as reflected by the term $P_{v} \beta_{m n}^{\left(A_{v}\right)} \varphi_{m}^{\left(A_{v}\right)}$ in the denominator of (4).

To obtain the SINR expression for BSC, note that, in the BSC operation, each MS communicates with more than one BS. Thus, the SINR expression for the BSC scheme involves an additional term compared to (4). For simplicity, we assume that the transmitting power of each cooperating BS is equally split among MSs that are involved in the cooperation, which can be achieved by a proper design of precoding matrices. ${ }^{2}$ Let $\mathbb{C}_{m}$ be the set of other MSs that engage in BSC with MS $m$.

\footnotetext{
${ }^{2}$ The precoding matrix design is beyond the scope of this paper. We refer interested readers to [23] and references therein.
}

Then, the received SINR (in the linear scale) at MS $m$ that uses subchannel $n$ is given in the form

$$
\begin{aligned}
& \operatorname{SINR}_{m n}(\mathrm{BSC}) \\
& =\frac{\frac{1}{1+\left|\mathbb{C}_{m}\right|}\left(P_{m} \beta_{m n}^{\left(A_{m}\right)} \varphi_{m}^{\left(A_{m}\right)}+\sum_{u \in \mathbb{C}_{m}} P_{u} \beta_{m n}^{\left(A_{u}\right)} \varphi_{m}^{\left(A_{u}\right)}\right)}{\sum_{v \in \mathbb{I}_{m}^{\prime}} P_{v} \beta_{m n}^{\left(A_{v}\right)} \varphi_{m}^{\left(A_{v}\right)}+N_{0}}
\end{aligned}
$$

where $\left|\mathbb{C}_{m}\right|$ is the cardinality of the set $\mathbb{C}_{m}$, and $\mathbb{I}_{m}^{\prime}$ is the set of interfering MSs for MS $m$. More specifically, the downlink transmission from the corresponding serving BS to the MS in the set $\mathbb{I}_{m}^{\prime}$ will cause interference to MS $m$.

\section{B. Multicell OFDMA Resource-Allocation Problem}

Here, we describe the multicell OFDMA channel allocation problem in the reuse-1 network. Let $\mathbf{Y}=\left[y_{m n}\right]$ be the channel assignment matrix whose entry $y_{m n}$ is equal to one if subchannel $n$ is assigned to MS $m$; otherwise, it is equal to zero. Then, the centralized multicell OFDMA resource-allocation problem can be formulated as follows.

$\mathbf{P}$. Find an assignment matrix, denoted by $\mathbf{Y}_{\mathrm{opt}}^{(P)}$, that maximizes the total capacity, i.e.,

$$
\mathbf{Y}_{\mathrm{opt}}^{(P)}=\arg \max _{\mathbf{Y}} \sum_{m=1}^{M} \sum_{n=1}^{N} \log _{2}\left(1+\operatorname{SINR}_{m n}\right) \cdot y_{m n}
$$

subject to the following two constraints:

$$
\begin{cases}\mathbf{C} 1: \forall n \in\{1,2, \ldots, N\}, & \text { if } y_{m^{\prime} n}=1, \text { then } y_{m n}=0 \\ \mathbf{C 2}: \forall m \in\{1,2, \ldots, M\}, & \text { for all } m \text { for which } A_{m}=\sum_{m}=\sum_{n=1}^{N} y_{m n} .\end{cases}
$$

Note that constraint $\mathbf{C} 1$ guarantees that a subchannel is used by at most one MS in each cell, i.e., no intracell interference. Constraint C2 states that the resource block demand ${ }^{3}$ of MS $m$, i.e., $R_{m}$, is met for all $m$. Note that constraints $\mathbf{C} 1$ and $\mathbf{C 2}$ should simultaneously be met. Thus, if all served MSs in a particular cell $l$ have an equal resource block demand of $R>1$, the number of served MSs in cell $l$ can be at most $N / R$, i.e., $M_{l} \leq N / R$.

Any attempt to solve Problem $\mathbf{P}$ would directly encounter two challenges. First, $\operatorname{SINR}_{m n}$ is unavailable before the actual resource allocation, because the interference for MS $m$ in subchannel $n$ depends on the utilization of subchannel $n$ by other MSs, which is unknown until $\mathbf{P}$ is solved. Second, Problem $\mathbf{P}$ is an NP-hard combinatorial optimization problem with nonlinear constraints. In other words, directly finding an optimal solution is computationally prohibitive, and no polynomial-time algorithm can optimally solve for $\mathbf{P}$.

In the next section, we address these challenges by proposing a new solution framework, where the obstacle of SINR mutual

\footnotetext{
${ }^{3}$ The number of subchannels will be equal to the throughput if all subchannels are statistically equal. Although the SINR of each subchannel will likely be statistically unequal in the multicell scenario, in light of the complex interdependency of SINR and the difficulty of obtaining exact statistics, we use C2 to approximate the throughput requirement.
} 
dependency is removed such that no exact SINR information is needed prior to the resource allocation, and the complexity is reduced by adopting heuristic algorithms.

\section{Proposed SOLUtion Framework}

\section{A. Graphic Approach}

The channel assignment problem in cellular and mesh networks has been studied in the context of multicoloring of a graph for decades (e.g., see [26]-[28]). In the traditional formulation, each node in a graph corresponds to a BS or an access point (AP) in the network to which channels are assigned. The edge that connects two nodes represents the potential cochannel interference in between, which typically corresponds to the geographical proximity of these two nodes. Then, the channel assignment problem becomes the node coloring problem, where two interfering nodes should not have the same color, i.e., use the same channel.

Our current problem, however, fundamentally differs from the conventional problem in three aspects. First, the traditional problem aims at minimizing the number of channels (i.e., colors) in use under the interference constraint, whereas we have a fixed and predetermined number of (sub)channels (i.e., colors) at disposal in the OFDMA network. In addition, complete avoidance of interference is often not physically possible in the reuse-1 deployment; thus, a proper compromise has to be considered. Second, nodes in the graph of our case should denote MSs rather than BSs, because channels are allocated to MSs in OFDMA networks. Furthermore, the location and movement of MSs will change the interference and, consequently, the graph. Third, the conventional graph of BSs contains edges that represent solely the cochannel interference, whereas the edge of our graph should be associated with a more general weight, because we incorporate technologies such as ICIC and BSC.

In the following discussion, we introduce the graph-based resource-allocation framework for multicell OFDMA. First, a method for constructing the interference graph is presented. Then, the two phases of the resource-allocation problem are conducted upon the interference graph.

\section{B. Interference Graph Construction}

The first step of the graphic approach to OFDMA resource allocation is to construct the interference graph that corresponds to the network topology. Consider an illustrative example with three BSs and five MSs, as shown in Fig. 2. Our objective is to construct a corresponding undirected interference graph, as shown in Fig. 3. In this graph, which is denoted by $G=(V, E)$, each node (from set $V$ ) represents an MS, and each edge (from set $E$ ) contains an integer "cost" or weight that characterizes the potential interference between two MSs. The weight of the edge $(a, b)$ is denoted by $w_{a b}$ and $w_{a b}=w_{b a}$.

We propose a method for determining the edge weight without accurate SINR measurements, because the measurement of SINR can be difficult in practice. The basic idea is to infer the interference intensity from the MS's geographic location. In particular, the weight associated with edge $(a, b)$ is determined based on the diversity set maintained at the BSs for MSs $a$ and $b$.

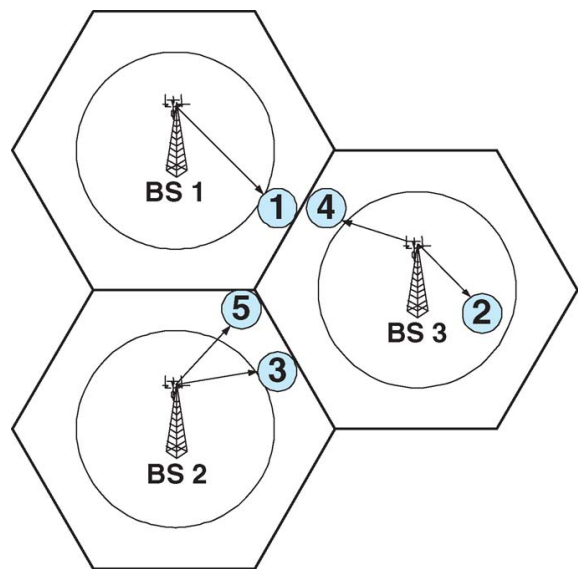

Fig. 2. Example of a multicell multiuser scenario.

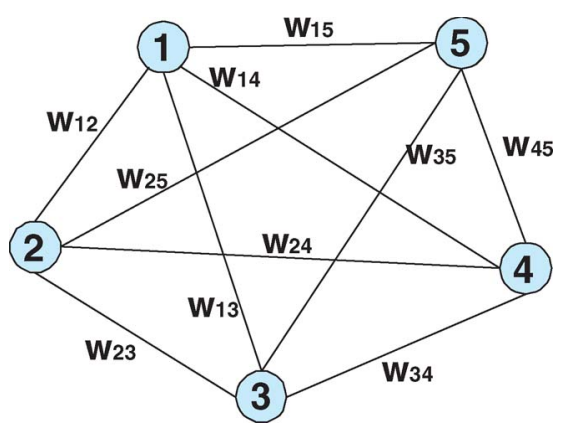

Fig. 3. Interference graph constructed for a multicell multiuser scenario.

TABLE I

DIVERSITY SET OF MSs IN FIG. 2

\begin{tabular}{l|l|l}
\hline & Anchor BS set & Neighbor BS set \\
\hline MS 1 & $\mathbb{A}_{1}=\{1\}$ & $\mathbb{B}_{1}=\{3\}$ \\
MS 2 & $\mathbb{A}_{2}=\{3\}$ & $\mathbb{B}_{2}=\phi$ \\
MS 3 & $\mathbb{A}_{3}=\{2\}$ & $\mathbb{B}_{3}=\{3\}$ \\
MS 4 & $\mathbb{A}_{4}=\{3\}$ & $\mathbb{B}_{4}=\{1\}$ \\
MS 5 & $\mathbb{A}_{5}=\{2\}$ & $\mathbb{B}_{5}=\{1,3\}$ \\
\hline
\end{tabular}

The diversity set contains useful geographical information that is related to the interference between MSs.

To cite an example, the diversity set for the scenario in Fig. 2 is given in Table I, where each row indicates the diversity set maintained for the corresponding MS. Each MS has an anchor BS (i.e., a serving BS) and, possibly, several neighbor BSs if it is located at the cell edge. For instance, MS 5 belongs to BS 2 but detects signals from BS 1 and BS 3 above the pathloss threshold such that the diversity set identifies them as the neighbor BSs. Thus, we have $\mathbb{A}_{5}=\{2\}$ and $\mathbb{B}_{5}=\{1,3\}$ for MS 5, as shown in Table I.

Given the diversity set information in Table I, we can infer the interference intensity between any two MSs as follows.

- Intracell interference. MS 2 and MS 4 have the same anchor BS and are, thus, within the same cell. Therefore, they will have intracell interference with each other.

- ICI and optional BSC. MS 1 and MS 4 each has an anchor BS that falls in each other's neighbor BS set. This case suggests that the downlink signal for MS 1 can reach MS 4, and vice versa. For this reason, transmission to MS 1 and MS 4 will cause ICI with both MS 1 and MS 4 . Meanwhile, this is also the precise condition under which 
BSC communication that involves MS 1 and MS 4 may be established.

- ICI but no optional BSC. MS 3 and MS 4 will have ICI, because the element of $\mathbb{A}_{4}$ is in the set $\mathbb{B}_{3}$ (i.e., the downlink signal for MS 4 from BS 3 will reach MS 3). However, BSC communication that involves MS 3 and MS 4 cannot be established, because the element of $\mathbb{A}_{3}$ is not in the set $\mathbb{B}_{4}$.

- No interference. MS 1 and MS 3 will not interfere with each other, because the anchor BS of neither MS is in the neighbor BS set of the other MS.

The aforementioned analysis is performed for every pair of nodes, followed by a proper weight assignment. There are six possible weight values between any two nodes, i.e.,

$$
w_{B}, w_{N}, w_{0}, w_{1}, w_{2}, w_{A}
$$

where $w_{B}, w_{N}$, and $w_{A}$ correspond to weights that are associated with BSC, no interference, and intracell interference, respectively, and $w_{0}, w_{1}$, and $w_{2}$ are ICI weights at various levels, depending on the geographic location of the two MSs. More specifically, the mutual ICI is the weakest if the two MSs are at the center of two adjacent cells (denoted by $w_{0}$ ), is medium if one MS is on the edge whereas the other is at the center of two adjacent cells (denoted by $w_{1}$ ), and is strongest if the two MSs are on the edge of two adjacent cells (denoted by $w_{2}$ ). The no-interference weight $w_{N}$ is set to zero to conform with the convention of "no edge" in the graph. The intracell interference weight $w_{A}$ should be assigned with a very large value, because the intracell interference must be avoided.

To support techniques such as BSC, which achieves interference management by allocating the same (rather than different) subchannel to interfering cell-edge MSs, we should assign the corresponding weight $w_{B}$ a very small value. Thus, in addition to the physical meaning of interference, where a bigger weight value represents stronger interference between MSs, the weight is also associated with the general meaning of functionality.

Overall, the six weight values can be ranked as

$$
\begin{aligned}
w_{B} \ll w_{N}(=0)<w_{0}<w_{1}<w_{2} \ll w_{A} \\
\left|w_{B}\right| \ll w_{A} .
\end{aligned}
$$

Note that BSC is an optional mechanism, whereas the intracell interference must be avoided; thus, we have the weight relationship in (10). More specifically, $w_{A}$ should significantly be large such that

$$
(L-1) w_{2}+2\left|w_{B}\right|<w_{A} .
$$

This condition will guarantee that the intracell interference can be avoided, as we will verify in Section V-B.

The complete algorithm for determining the edge weight is summarized in Table II. In Table II, the anchor BS of MS $a$ and MS $b$ are first examined.

Step 1) If they are the same, the weight decision can directly be made, i.e., we assign $w_{a b}$ with value $w_{A}$.

Step 2) If they are not the same, then further procedures are needed. In particular, depending on whether the anchor BS of MS $a$ is in the neighbor BS set of
TABLE II

Algorithm For Determining the Weight of the EdGE $(a, b)$

Initialize: If MSs $a$ and $b$ are configured to perform BSC whenever feasible, $\alpha_{B}=1$; otherwise, $\alpha_{B}=0$. If MS $a$ (or $b$ ) is in cell edge, $\gamma_{a}\left(\right.$ or $\left.\gamma_{b}\right)=1$; otherwise, $\gamma_{a}$ (or $\left.\gamma_{b}\right)=0 . I_{B S C}=0$.

1. If $\mathbb{A}_{a} \cap \mathbb{A}_{b} \neq \phi$,

2. If $\mathbb{A}_{a} \cap \mathbb{B}_{b} \neq \phi$,

$$
w_{a b}=w_{A} \text {. Go to } 6 .
$$

Else,

$$
\begin{aligned}
& w_{a b}^{(1)}=w_{\gamma_{a}+\gamma_{b}} \\
& I_{B S C}=I_{B S C}+1 .
\end{aligned}
$$

3. If $\mathbb{A}_{b} \cap \mathbb{B}_{a} \neq \phi$,

$$
w_{a b}^{(1)}=w_{N} \text {. }
$$

$w_{a b}^{(2)}=w_{\gamma_{a}+\gamma_{b}}$

$I_{B S C}^{a b}=I_{B S C}+1$

Else,

$$
w_{a b}^{(2)}=w_{N} .
$$

4. If $I_{B S C}=2$ and $\alpha_{B}=1$,

5. $w_{a b}=\max \left(w^{(1)}, w_{a b} w^{(2)}\right) w_{B}$. Go to 6

6. Output $w_{a b}$.

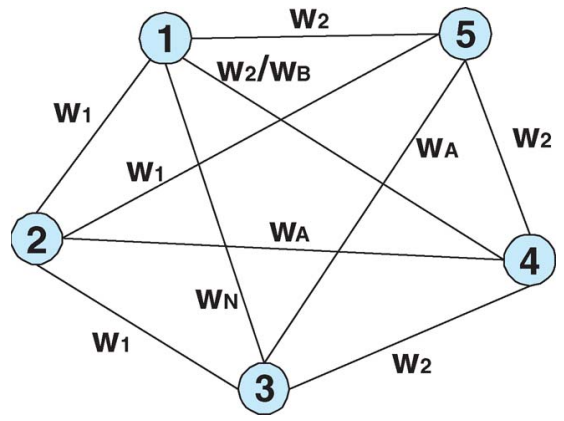

Fig. 4. Interference graph for the scenario given in Fig. 2.

MS $b$, the temporary weight $\left(w_{0}, w_{1}, w_{2}\right)$ or $w_{N}$ is accordingly assigned to $w_{a b}^{(1)}$.

Step 3) Depending on whether the anchor BS of MS $b$ is in the neighbor BS set of MS $a$, the temporary weight $\left(w_{0}, w_{1}, w_{2}\right)$ or $w_{N}$ is accordingly assigned to $w_{a b}^{(2)}$.

Step 4) If the anchor BS of each MS is in the neighbor BS set of the other MS, BSC may be performed. If the system determines that BSC will be used for these two MSs, assign $w_{B}$.

Step 5) If the case is otherwise, $\operatorname{assign} \max \left(w_{a b}^{(1)}, w_{a b}^{(2)}\right)$.

The resulting interference graph with an assigned weight for Fig. 2 is illustrated in Fig. 4, where there can be two possible weights for some edge, depending on the actual configuration of MSs. It is assumed that the configuration choice is predetermined before the allocation process. For example, if MS 1 and MS 4 are both preconfigured to perform BSC whenever feasible, edge $(1,4)$ will be assigned with weight $w_{B}$; otherwise, it will be assigned with weight $w_{2}$. BSC cannot be established between the remaining node pairs in the graph; thus, they are left with only one option, and proper weights are assigned according to the algorithm in Table II.

Once the interference graph is constructed, our solution to the resource-allocation problem follows a two-phase approach on the graph: 1) the coarse-scale ICI management and 2) the fine-scale channel assignment. These phases are described as follows. 


\section{First Phase: Interference Management}

There is a close relationship between the MAX $k$-CUT problem [29] in a general graph theory and the interference management problem in OFDMA networks. In the graph theory, a cut is a partition of the vertices of the graph into multiple sets or clusters. The size of a cut is the total number of edges that cross the cut. In our weighted graphs, the size of the cut is the sum of weights of the edges that cross the cut. A cut is maximal $(\max )$ if the size of the cut is not smaller than the size of any other cut. By generalizing a cut to $k$ cuts, the MAX $k$-CUT problem is to find a set of $k$ cuts that are not smaller in size than any other $k$ cuts.

Given $N$ subchannels and $M$ MSs, our interference management problem is a MAX $N$-CUT problem on the interference graph and is formally stated as follows.

P1. Given an interference graph $G=(V, E)$ with $M$ nodes and edge weight $w_{a b}$ for each edge $(a, b)$, find a partition of the graph into $N(N \geq 2)$ disjoint clusters $\mathbb{R}_{i}, i=$ $1, \ldots, N$, such that $\bigcup_{i=1}^{N} \mathbb{R}_{i}=V$ and $\sum_{a \in \mathbb{R}_{i}, b \in \mathbb{R}_{j}, i<j} w_{a b}$ is maximized.

Here, each cluster corresponds to a subchannel. Nodes (or MSs) in the same cluster will be assigned with the same subchannel. With the goal of maximizing the intercluster edge weight in Problem P1, the result will tend to place strong interferers into different clusters or, equivalently, separate them on different subchannels, which helps in reducing ICI. If BSC is supported, the clustering result will tend to place BSC-feasible MSs into the same cluster to allow cooperation due to the small BSC weight value $w_{B}$.

Both constraints $\mathbf{C} 1$ and $\mathbf{C 2}$ of Problem $\mathbf{P}$ in (7) are readily addressed in this graphic framework. The very large intracell interference weight $w_{A}$ will ensure the complete avoidance of intracell interference as stated in C1. To meet the resource block demand $R_{m}=\sum_{n=1}^{N} y_{m n}$ in $\mathbf{C 2}$, we can duplicate the node that corresponds to MS $m$ for $R_{m}$ times in the interference graph and assign a very large weight (e.g., $w_{A}$ ) to edges that connect these duplicate nodes. This way, these duplicate nodes will be placed into different clusters, and consequently, $R_{m}$ subchannels will be assigned to MS $m$ to meet the resource block demand.

\section{Second Phase: Channel Assignment}

After the first-phase partition, MSs are grouped into $N$ clusters for subchannel allocation. In the second phase, we should decide which subchannel will be allocated to which cluster. Among $N$ ! possible subchannel assignment choices, the second-phase assignment aims at finding the choice that best leverages the instantaneous channel quality. The problem is formulated as follows.

P2. Let $\mathbb{J}$ be the set formed by $N$ ! valid subchannel assignment choices after the first phase. Find an assignment matrix $\mathbf{Y}_{\mathrm{opt}}^{(P 2)}$ such that

$$
\mathbf{Y}_{\mathrm{opt}}^{(P 2)}=\arg \max _{\mathbf{Y} \in \mathbb{J}} \sum_{m=1}^{M} \sum_{n=1}^{N} \log _{2}\left(1+\mathrm{SNR}_{m n}\right) \cdot y_{m n}
$$

TABLE III

Heuristic Algorithm A1 for Solving Problem P1

Initialize: Let $W_{i}=\sum_{u \in \mathbb{R}_{i}, v \in \mathbb{R}_{i}} w_{u v}$ be the intracluster weight of cluster $\mathbb{R}_{i}, i=1, \ldots, N$. Initially, since clusters have not yet been assigned with nodes, $W_{i}=0, \forall i=1, \ldots, N$.

1. Arbitrarily order $M$ nodes.

2. Assign the first $N$ nodes to $N$ clusters, one in each cluster

3 . Take the next node from the ordered nodes. Let this node be node $m$. Let $W_{i}^{a}$ be the increased intracluster weight to cluster $\mathbb{R}_{i}$ if node $m$ is assigned to cluster $\mathbb{R}_{i}$. Specifically, $W_{i}^{a}$ is the sum of edge weights between node $m$ and all nodes that have already been assigned to cluster $\mathbb{R}_{i}$, i.e., $W_{i}^{a}=\sum_{v \in \mathbb{R}_{i}} w_{m v}$. Collect $W_{i}^{a}$ for all $i=$ $1, \ldots, N$

4. Assign node $m$ to cluster $\mathbb{R}_{i^{*}}$, where $i^{*}=\arg \min _{i} W_{i}^{a}$. If there is more than one minimum, break the tie randomly.

5. Update the intracluster weight of cluster $\mathbb{R}_{i^{*}}$ such that $W_{i^{*}}=W_{i^{*}}+$ $W_{i^{*}}^{a}$

6. Repeat Steps 3-5 for all nodes.

where $\mathrm{SNR}_{m n}$ is the instantaneous channel quality between MS $m$ and its serving BS on subchannel $n$, which is proportional to $\beta_{m n}^{\left(A_{m}\right)}$.

Note that ICI is dealt with in the first phase; thus, the second phase considers only SNR, which avoids the interdependency issue.

\section{Proposed Algorithms}

In this section, we present heuristic algorithms for the two phases of the resource-allocation problem in Section IV.

\section{A. Heuristic Algorithm A1 for the First Phase}

Problem $\mathbf{P} 1$ is an NP-hard problem for a graph with a large number of nodes [30]. That is, the optimal solution for P1 is computationally prohibitive for large graphs. Consequently, we apply the simple heuristic algorithm in [29] on Problem $\mathbf{P 1}$, which can efficiently produce an approximate solution. It is proven in [29] that, given that all weights in the graph are nonnegative integers, the heuristic algorithm achieves an absolute ratio of $(1-1 / k)$ for a general MAX $k$-CUT problem. That is, the algorithm can yield a clustering in which the intercluster weight sum is at least $(1-1 / k)$ times the optimal cut. In our case of $k=N$, with some weight being negative, the algorithm can produce $(1-1 / N)$ times the optimal solution on the shifted version of weights, i.e., after the absolute value of the most negative weight is added to all weight values to make all weights nonnegative.

The idea of the algorithm is to iteratively assign nodes to the cluster such that, at each step, the increased intracluster weight is minimized. The detailed description of the algorithm is given in Table III for a practical scenario with $M>N$. The clustering problem becomes trivial when $M \leq N$, because the amount of OFDMA resource that is available for allocation (i.e., $N$ ) is greater than or equal to the resource needed by the MSs (i.e., $M$ number of MSs) such that we can just give each MS its own subchannel. In this case, the algorithm terminates at Step 2) with the optimal solution.

If $M>N$, the algorithm proceeds by the following steps.

Steps 1) and 2) Assign $N$ arbitrarily chosen nodes to $N$ clusters, one in each cluster. 


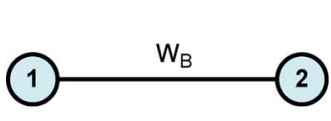

(a)

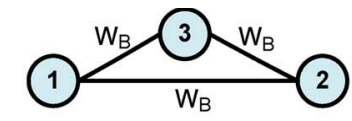

(b)

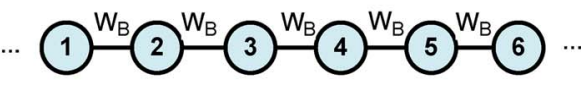

(c)

Fig. 5. Possible BSC occurrences. (a) BSC clique of two. (b) BSC clique of three. (c) BSC cascade.

Steps 3) and 4) The remaining $M-N$ nodes are iteratively assigned at each step to the cluster for which the increased intracluster weight is minimized.

Step 5) Once the new assignment is done, the intracluster weight of the cluster is updated.

Step 6) The iteration repeats until all nodes are assigned into a cluster.

The complexity of this heuristic method is proportional to the sum of the number of edges, nodes, and clusters in the graph. For our particular case with $M$ nodes and $N$ clusters, this heuristic method is of complexity $O\left(M^{2} / 2+M / 2+N\right)$.

\section{B. Properties of Algorithm A1}

Some discussions on the properties of the clustering algorithm A1 are presented here. The objective is to show that algorithm A1, along with the weight assignment in Section IV-B, will produce desirable results.

Property 1 (BSC-Weight Assignment): An MS node can be connected by a BSC $\left(w_{B}\right)$ weighted edge with other MSs in at most two neighbor cells.

Proof of Property 1: First, we note that the BSC weight is assigned to the edge between two MSs when each MS's anchor BS is in each other MS's neighbor BS set (see Section IV-B). Second, by the property of forming the diversity set in Section II-B, an MS has at most two neighbor BSs in its diversity set (which is denoted here, for convenience, as BS $a$ and BS $b$ ). Thus, an MS will have a BSC-weighted edge only with MSs that are served by either BS $a$ or BS $b$.

Property 2 (Intracell Interference Avoidance): Any two intracell MSs will be placed into two different clusters by algorithm A1.

Proof of Property 2: Denote any two intracell MSs by MS $a$ and MS $b$. According to our weight assignment rule, $w_{a b}=w_{b a}=w_{A}$. If, at Step 2) of A1, MS $a$ and MS $b$ are assigned into different clusters, we are done. If not, MS $a$ and MS $b$ are clustered at different iterations. Assume, without loss of generality, that MS $a$ is clustered before MS $b$ is clustered. Consider, at some iteration, that MS $b$ is selected for clustering decision, MS $a$ has already been placed in cluster $\mathbb{R}_{i}$, and all previous iterations did not place intracell MSs, if any, into the same cluster. We intend to show that the current iteration will not place intracell MSs into the same cluster either, or specifically, MS $b$ will be clustered into a different cluster and not in $\mathbb{R}_{i}$ at this iteration to complete the proof.

The clustering decision for MS $b$ starts with Step 3) of A1, where the increased intracluster weight is calculated for all clusters, i.e.,

$$
W_{i^{\prime}}^{a}=\sum_{v \in \mathbb{R}_{i^{\prime}}} w_{b v}, \quad i^{\prime}=1, \ldots, N
$$

Note that the summing terms in calculating $W_{i}^{a}$ contain one $w_{A}$, because MS $a$ is in cluster $\mathbb{R}_{i}$, and at most two $w_{B} \mathrm{~S}$ due to Property 1 and the fact that intracell MSs, if any up to this iteration, are in different clusters. Thus, we have $W_{i}^{a} \geq$ $w_{A}+2 w_{B}$. On the other hand, the number of intracell MSs in any cell $l$ is not greater than the number of subchannels, i.e., $M_{l} \leq N$; thus, MS $b$ is connected by at most $N-1$ intracell $\left(w_{A}\right)$ weighted edges. We have $N$ clusters; thus, there must exist some cluster $\mathbb{R}_{j}, j \neq i$, for which the summing terms in calculating $W_{j}^{a}$ do not include $w_{A} . w_{2}$ is the next largest weight to $w_{A}$, and the maximum number of nodes that are currently in cluster $\mathbb{R}_{j}$ is $L-1$ (we have $L$ cells); thus, we have $W_{j}^{a} \leq(L-1) w_{2}$. Due to the fact that $w_{A}$ is significantly large and in particular, because of (11), we are led to $W_{j}^{a}<W_{i}^{a}$. Thus, Step 4) of A1 will place MS $b$ into a different cluster and not in $\mathbb{R}_{i}$, which completes the proof.

Property 3 (Feasibility of BSC): Define the number of distinct MSs and BSs that are involved in the same BSC event on a particular subchannel to be $M_{\mathrm{BSC}}$ and $L_{\mathrm{BSC}}$, respectively. A BSC event is "feasible" if $M_{\mathrm{BSC}} \leq L_{\mathrm{BSC}}$, i.e., the virtual operation of BSC can realistically be supported. Algorithm A1 will always produce feasible BSC.

Proof of Property 3: Assume that $M_{\mathrm{BSC}}>L_{\mathrm{BSC}}$. BSC is established between BSs and MSs that are served by these BSs; thus, there must be at least two MSs that are served by the same BS if $M_{\mathrm{BSC}}>L_{\mathrm{BSC}}$, i.e., they are intracell MSs. By Property 2, these two MSs will be placed into different clusters. However, by definition, all $M_{\mathrm{BSC}}$ MSs are involved in the same BSC event and, therefore, are placed in the same cluster. By contradiction, we have $M_{\mathrm{BSC}} \leq L_{\mathrm{BSC}}$.

Note that some BSC operations may be feasible but not necessarily efficient. In particular, a BSC operation that involves many geographically far BSs is, although feasible by Property 3, not an efficient way of leveraging the advantage of performing BSC. In the following discussion, we aim at showing that, by first stating a property that directly follows from our interference graph construction method and next proposing a method of treating a complex BSC scenario, algorithm A1 will produce a BSC event that involves at most three (geographically close) BSs and three MSs.

Property 4 (Clustering of BSC): Algorithm A1 will yield a clustering result where an MS node is connected by at most two BSC-weighted edges that are intracluster edges (i.e., edges with two ends within the same cluster).

Proof of Property 4: This result directly follows from Properties 1 and 2.

Following the result in Property 4, BSC events may occur in one of the three possible scenarios in Fig. 5. All MS nodes in each scenario are placed in the same cluster for BSC to take place, and each MS node is connected by at most two BSCweighted edges due to Property 4. The scenarios in Fig. 5(a) 


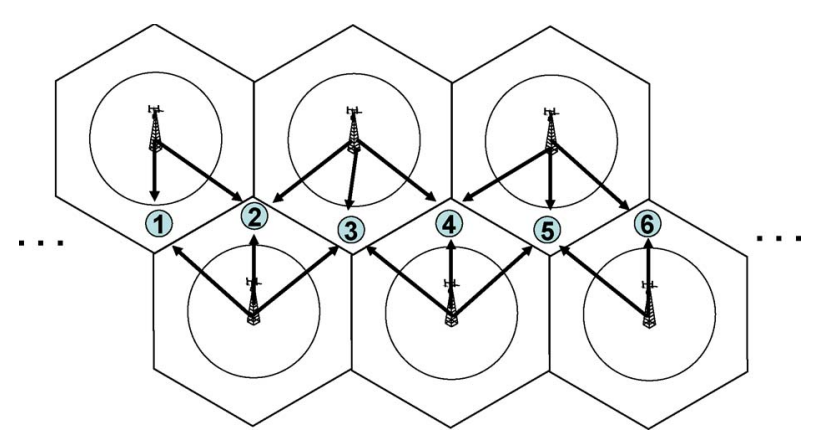

Fig. 6. Cascade topology for BSC operation.

and (b) are "BSC cliques," 4 and a proper degree of BSC will accordingly be performed (i.e., two-MS, two-BS or three-MS, three-BS BSC). A BSC clique can be of any size not greater than three due to Property 4.

The third scenario where BSC events might occur is the "BSC cascade" scenario in Fig. 5(c). Edges that are not shown here are not BSC weighted. Note that two end nodes in this scenario do not link (by a BSC-weighted edge), unless the number of nodes is less than or equal to three, which degenerates to the scenarios in Fig. 5(a) and (b). ${ }^{5}$ In other words, in the cascade scenario, no three MSs are pairwise connected by BSC weights (i.e., no BSC cliques of three), but any MS, except for the end nodes, is connected by two BSC-weighted edges with another two MSs. One possible topology that corresponds to Fig. 5(c) is drawn in Fig. 6. It is shown that, due to the geographical location and the diversity set, MS 1 and MS 3 do not have a BSC-weighted edge in between, but each of them has a BSC-weighted edge with MS 2. The solid arrows in Fig. 6 show the downlink transmission in a potential BSC operation in the cascade setting. However, as shown in Fig. 6, different BSC transmissions may cause interference to each other (e.g., consider a BSC event among MS 1, MS 2, and MS 3, and another BSC event among MS 2, MS 3, and MS 4). To address this issue, we propose a method of breaking up the cascade into multiple BSC cliques of two when algorithm A1 produces a BSC cascade.

Treating the BSC Cascade: It is assumed that, after the clustering achieved by algorithm A1, the RNC can identify the physical challenge of performing some BSC events that A1 yielded, e.g., the cascade scenario. Then, a negotiation will take place between BSs and MSs such that the edge weight between some MSs is changed from BSC weight $\left(w_{B}\right)$ to ICI weight $\left(w_{0}, w_{1}\right.$, or $\left.w_{2}\right)$ to break up the cascade. For example, if the BSC weights between nodes 2 and 3 and nodes 4 and 5 in Fig. 5(c) are changed to ICI weights, the cascade is broken up into three BSC cliques of two. In general, for a cascade that contains $M_{\text {cas }}$ distinct MSs, where $M_{\text {cas }} \geq 3,\left\lfloor\left(M_{\text {cas }}-1\right) / 2\right\rfloor$ changes of weight are needed. Then, algorithm $\mathbf{A} 1$ is run again with the new set of weights.

\footnotetext{
${ }^{4} \mathrm{~A}$ BSC clique is a graph in which every node is connected by a BSCweighted edge to every other node.

${ }^{5}$ It can easily be verified that the scenario of four or more nodes that form in a circle is not possible due to the hexagonal network topology and the diversity set property used to determine BSC relations.
}

TABLE IV

Heuristic Algorithm A2 for Solving Problem P2

Initialize: Let $\Phi=\{1, \ldots, N\}$ be the subchannel pool. Order $N$ clusters in size from the smallest to the largest (break the tie arbitrarily).

1. Examine clusters in order, one at a time. Let the selected cluster be $\mathbb{R}_{i}$. Calculate the sum capacity $T_{n}=\sum_{m \in \mathbb{R}_{i}} \log _{2}\left(1+\mathrm{SNR}_{m n}\right)$ for all $n \in \Phi$.

2. Assign subchannel $n^{*}$ to cluster $\mathbb{R}_{i}$, where $n^{*}=\arg \max _{n} T_{n}$.

3. Update subchannel pool to exclude $n^{*}$, i.e., $\Phi=\Phi \backslash\left\{n^{*}\right\}$.

4. Repeat Steps 1-3 for all clusters.

Note that the BSC cascade scenario is, however, rare in practice. In fact, the cascade scenario will occur only when all of the following conditions are simultaneously met.

1) There must be some MSs within the three-cell overlapping coverage area. For a long cascade to happen, there must be a "string" of MSs, each located in a different (and nearby) three-cell overlapping area.

2) Some MSs in the cascade may have other BSC choices ("partners") that will not result in any cascade. Among these choices, algorithm $\mathbf{A} 1$ chooses one that produces a cascade.

3) Step 2) of algorithm A1 does not initially break up the cascade by assigning some MSs in the cascade into different clusters.

The likelihood that Condition 1 will be met is small in a typically large cell coverage area with a relatively small threecell overlapping area, as we will verify in the simulation. The likelihood that Condition 2 will be met is reduced when more MSs in the potential cascade have some other BSC choices such that a simultaneous selection of particular BSC pairs to result in a cascade is less likely. Although whether Condition 3 will be met or not is totally random, the likelihood is, in general, reduced in lighter load networks (i.e., smaller $M$ ).

After the aforementioned discussion on the possible BSC occurrences, we are led to the following final property.

Property 5 (Degree of $B S C$ ): Algorithm A1 will produce BSC events that involve at most three MSs and their corresponding three (geographically close) anchor BSs. That is, BSC operation will be both feasible and efficient.

Proof of Property 5: This result directly follows from the discussion on the aforementioned possible BSC scenarios. In particular, for BSC cliques of two, BSC cliques of three, and BSC cascade scenarios after properly treated, it is easily shown that the produced BSC events will involve at most three MSs and the corresponding three anchor BSs.

\section{Heuristic Algorithm A2 for the Second Phase}

An exhaustive search through all $N$ ! choices for solving Problem P2 is also computationally infeasible. We propose a heuristic suboptimal algorithm that iteratively assigns subchannels to clusters, as described in Table IV. We call this method max-SNR channel assignment.

In Table IV, the initial subchannel pool is $\{1, \ldots, N\} . N$ clusters are ordered from small to large in size in terms of the number of nodes contained in the cluster, with ties broken 
TABLE V

SIMULATION SETUP

\begin{tabular}{ll}
\hline Cell Parameters & \\
\hline Number of Cells, $L$ & 19, wrap-around \\
Cell Radius & $750 \mathrm{~m}$ \\
Cell-center Radius & $500 \mathrm{~m}$ \\
Intercell Distance Ratio $^{a}, \rho$ & 0.9 \\
Antennas $N_{T}, N_{R}$ & 4,2 \\
Frequency Reuse Factor & 1 \\
\hline OFDMA Parameters & \\
\hline FFT size & 1024 \\
Carrier Frequency & $2.5 \mathrm{GHz}$ \\
Sampling Frequency & $11.2 \mathrm{MHz}$ \\
Number of Subchannels, $N$ & 30 \\
Number of Subcarriers Per Subchannel & 28 \\
DL Permutation Type & PUSC \\
\hline Channel Model & \\
\hline Path Loss (dB) & $130.62+37.6 * \log _{10}(d)$, \\
& $(d$ in km $)$ \\
Fast Fading & ITU Pedestrian B \\
\hline
\end{tabular}

Power Control Parameters

Cell-center Trans. Power, $P_{0}$

Cell-edge Trans. Power, $P_{1}$

Thermal Noise Power, $N_{0}$

$40 \mathrm{dBm}$

$46 \mathrm{dBm}$

$-119 \mathrm{dBm}$

\footnotetext{
${ }^{a}$ Intercell distance may be shortened or expanded to control cell overlapping area. The ratio shown here is relative to the hexagonal back-to-back cell deployment.
}

arbitrarily. Smaller clusters are first examined, because the choice of subchannels makes more impact to smaller clusters.

Steps 1) and 2) For each particular cluster, the subchannel for which the sum capacity is maximum is assigned to this cluster.

Step 3) The procedure continues on the next cluster with the subchannel pool of one less entry, i.e., the subchannel that has already been assigned is removed from the pool.

Step 4) The iteration repeats until all clusters are assigned with one subchannel.

This heuristic method that iteratively assigns subchannels to clusters is of complexity $O\left(N^{2}\right)$.

One alternative method called random channel assignment can also be used here to solve the second-phase problem. In this method, one assignment out of $N$ ! choices is randomly picked as the solution. The complexity of this random assignment method is $O(1)$. However, the performance of the random channel assignment may not be as good as that of the previously described heuristic method, because it does not consider channel condition when performing the channel assignment.

\section{Simulation Results}

In this section, we study the performance of the proposed schemes by computer simulation. The simulation setup closely follows the IEEE $802.16 \mathrm{~m}$ evaluation methodology [31] and is summarized in Table V.

The five schemes that will be investigated and compared are shown in Table VI. ICI-blind is the traditional OFDMA scheme where no ICI-aware mechanism is employed; i.e., each cell independently performs its own channel allocation without
TABLE VI

FIVE TEST SCHEMES

\begin{tabular}{l|ll}
\hline Scheme & $\begin{array}{l}\text { First Phase: } \\
\text { Interference Management }\end{array}$ & $\begin{array}{l}\text { Second Phase: } \\
\text { Channel Assignment }\end{array}$ \\
\hline ICI-blind & no ICI consideration & random \\
ICIC1 & ICIC & random \\
ICIC2 & ICIC & max-SNR \\
BSC1 & ICIC+BSC & random \\
BSC2 & ICIC+BSC & max-SNR \\
\hline
\end{tabular}

intracell interference. The rest are our proposed schemes, which differ in the interference management mechanism in the first phase (i.e., ICIC or ICIC + BSC) and in the second phase (i.e., random or max-SNR assignments). The graph edge weights are chosen to be

$$
\left(w_{B}, w_{N}, w_{0}, w_{1}, w_{2}, w_{A}\right)=\left(-10^{3}, 0,50,100,200,10^{5}\right) .
$$

It is worthwhile to note that the performance of our proposed graph-based scheme is not sensitive to the chosen weight values, which is another highly desirable feature of this solution approach. Indeed, as revealed by the simulation, as long as the interrelationship of weights in (9)-(11) is respected, a small variation in the weight does not change the final channel assignment decision that the proposed algorithm yielded.

Fig. 7(a)-(c) show the cumulative distribution function (cdf) of SINR for five test schemes under different traffic load conditions (with 25, 15, and five uniformly distributed MSs per cell, respectively). It is evident that both ICIC and BSC schemes have a remarkable improvement on the SINR performance compared with the ICI-blind scheme. This result demonstrates the effectiveness of the proposed ICI-reduction schemes. We also see a higher additional gain of ICIC2 (BSC2) compared with ICIC1 (BSC1) in lower load conditions, because interference dominates in higher load conditions, and the channelaware resource assignment makes a diminishing impact in the second phase. In addition, due to fewer interferers, the average SINR increases for all schemes as the traffic load decreases, as shown in Fig. 7(a)-(c).

The average SINR gains of the proposed schemes with respect to the ICI-blind scheme under various traffic loads are compared in Fig. 8. Several observations can be drawn based on this figure. First, as we have previously discussed, we see a more significant gain in ICIC2 and BSC2 in low-load situations. Second, the ICIC gain significantly drops in very high load situations, because the inevitable channel collision in the presence of a large number of MSs has rendered the ICIC strategy ineffective, if still feasible at all. In contrast, BSC retains the gain as the traffic load increases and experiences only minor degradation in very-high-load situations. This instance is explained by the fact that, although a high load creates a high-interference environment, it also creates more BSC opportunities. BSC can only be established among MSs that are "geographically fitting"; thus, a higher load increases the number of MSs that can be engaged in BSC and, consequently, the number of actual events of BSC. This effect counteracts the degradation caused by more interferers.

The effect of unequal cell loading on the SINR performance is examined in Fig. 9. We simulate an unequal-load scenario with nine randomly selected cells of $25 \mathrm{MSs}$, nine cells of five 


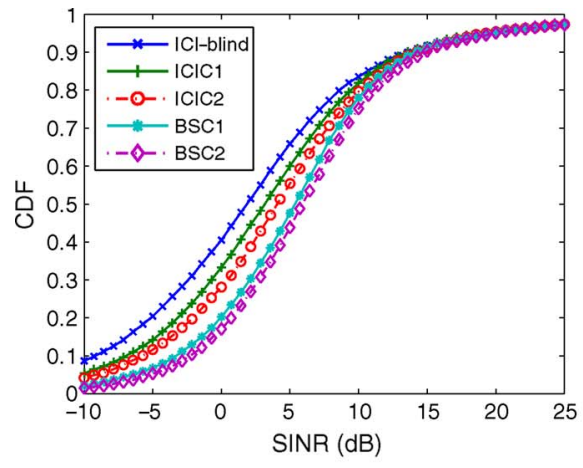

(a)

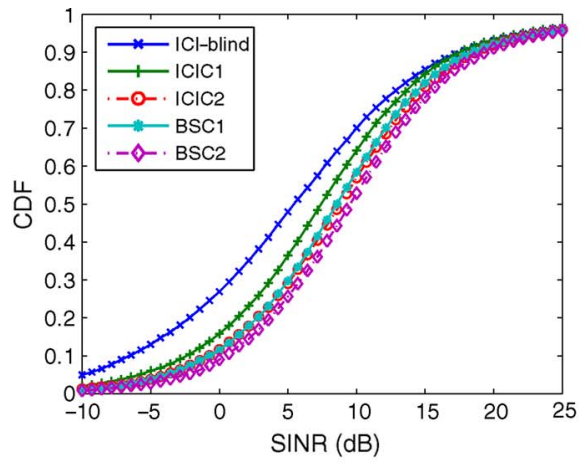

(b)

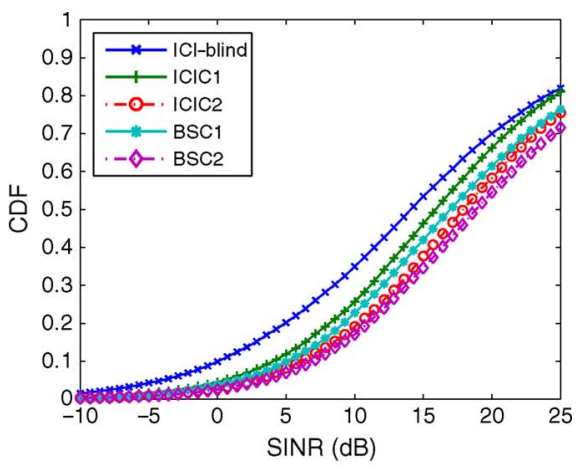

(c)

Fig. 7. SINR distribution for different traffic load conditions. (a) Twenty-five MSs per cell. (b) Fifteen MSs per cell. (c) Five MSs per cell.

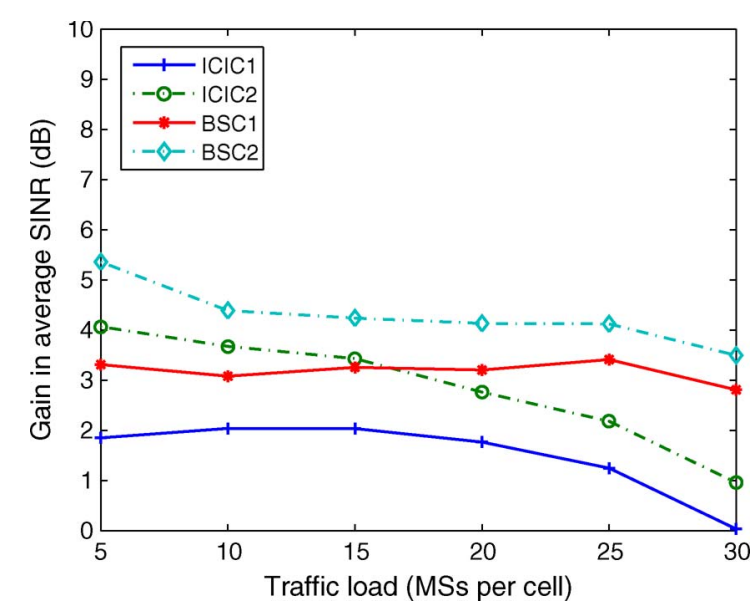

Fig. 8. Average SINR gains with respect to the ICI-blind scheme under different traffic loads.

MSs, and one cell of 15 MSs to make an average of 15 MSs per cell in our 19-cell simulator. Cell-specific SINR performance is shown in Fig. 9(a) for heavy-load cells (i.e., 25 MSs) and in Fig. 9(b) for light-load cells (i.e., five MSs). We see that both figures have performance close to the uniform 15-MSper-cell scenario in Fig. 7(b). However, heavy-load cells achieve slightly better performance than light-load ones due to the slightly lighter traffic in the neighboring cells "seen" by a heavy load cell, which leads to less ICI and better performance.

Fig. 10 shows the effect of downlink PC on the SINR performance. We compare the case with PC (i.e., $P_{0}=40 \mathrm{dBm}$ and $P_{1}=46 \mathrm{dBm}$ ) and without PC (i.e., $P_{0}=P_{1}=46 \mathrm{dBm}$ ). It is observed in Fig. 10(a) that PC improves the low-SINR performance, because the reduced transmission power to cell-center MSs causes less interference to neighboring cells. This instance inevitably compromises the SINR performance of the MSs in the cell center, as revealed in Fig. 10(b), because a reduction in transmission power for cell-center users will decrease their received signal strength. Nevertheless, these figures still justify the use of power control from the system capacity point of view, because the performance improvement in the low-SINR regime is far more important than the minor SINR degradation in the high-SINR regime. Moreover, a reasonable degree of SINR deterioration for these cell-center users normally does not cause an immediate drop in their modulation-coding-scheme level and, therefore, will not entail a decrease in the throughput perceived by these MSs in the cell center.
Fig. 11(a) compares two permutation schemes, i.e., PUSC and AMC, which are defined in the IEEE 802.16e standard. PUSC scrambles subcarriers before grouping them into a subchannel, and thus, the quality of subchannels is expected to statistically be alike. Therefore, performing the second-phase task yields a smaller gain. In contrast, AMC maps physically contiguous subcarriers to a logical subchannel. Thus, the disparity between subchannels is anticipated to be higher due to frequency-selective fading across subcarriers. This case contributes to a higher performance gain associated with ICIC2 and BSC2 with AMC permutation.

In real-world cellular deployment, it is desirable to balance between the BS placement density and QoS. If very few BSs are deployed in a given region, some areas are not covered. On the other hand, an overly dense BS installation will not only result in increased deployment cost but also lead to aggravated ICI. We examine this issue in Fig. 11(b) by adjusting a parameter called intercell distance ratio $\rho$, as defined in Table V. Three different cell deployment schemes (i.e., $\rho=1,0.9$, and 0.8 ) are compared. When cells are closer to each other (i.e., smaller $\rho$ ), ICI is higher, and consequently, the employment of an ICI management scheme is more beneficial, as revealed by the higher gain in Fig. 11(b). In addition, smaller $\rho$ also provides more coverage overlapping areas in which BSC can be performed by MSs in these areas. In other words, denser deployment of cells provides an extra advantage for the BSC scheme, which accounts for the 6-dB gain of BSC2 when $\rho=0.8$.

Fig. 12 draws the contour plot of the SINR level in a cell plane, as shown by the circle to represent the coverage. The "heights" in the plot represent the SINR in decibels. Each concentric circle shows an SINR value after averaging over several MSs at that particular distance away from the BS. Three schemes are compared under the same traffic load of $25 \mathrm{MSs}$ per cell. As shown in Fig. 12(a)-(c), ICI-blind, ICIC1, and BSC1 achieve equally high SINR values in the cell center but differ in the cell edge. In particular, the cell-edge SINR value is the lowest for ICI-blind, medium for ICIC1, and the highest for BSC1. This figure shows that ICI management is particularly helpful to cell-edge MSs. In addition, BSC1 observes an SINR increase toward the cell edge because of the use of BSC in the cell edge. This figure also reveals the geographical relationship of the SINR distribution.

Fig. 13 shows the spectrum usage in specific cell-edge areas to demonstrate how our proposed algorithms achieve the 


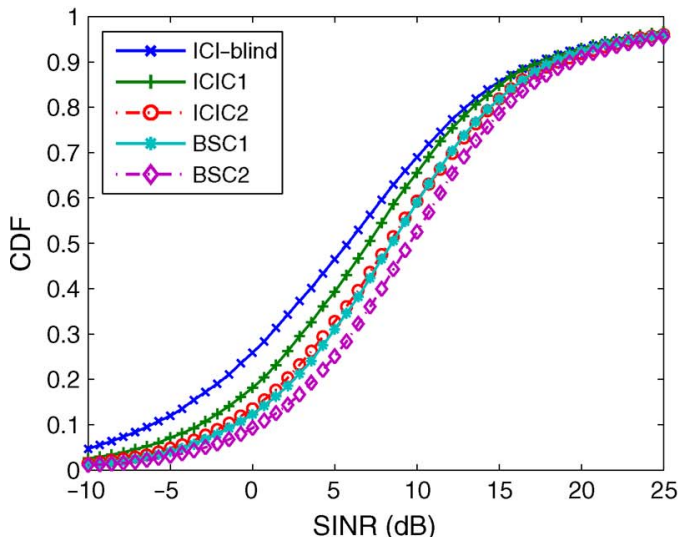

(a)

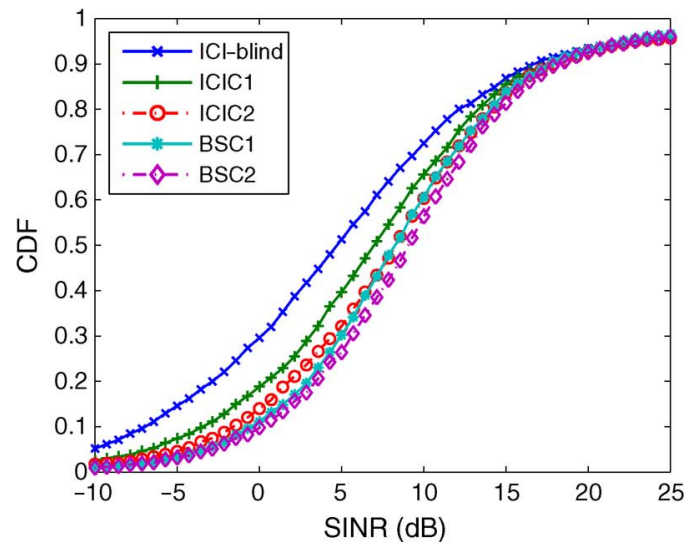

(b)

Fig. 9. Cell-specific SINR distribution under an unequal-cell-load scenario (i.e., nine cells of 25 MSs, nine cells of five MSs, and one cell of 15 MSs). (a) Heavy-load cells (i.e., $25 \mathrm{MSs}$ ). (b) Light-load cells (i.e., five MSs).

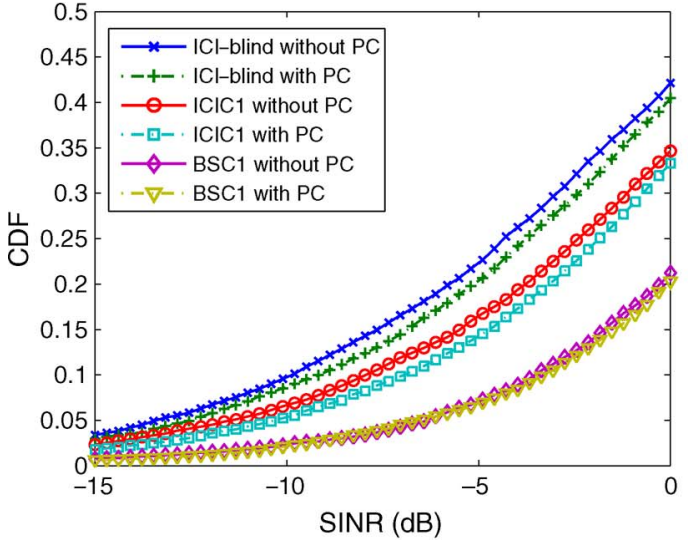

(a)

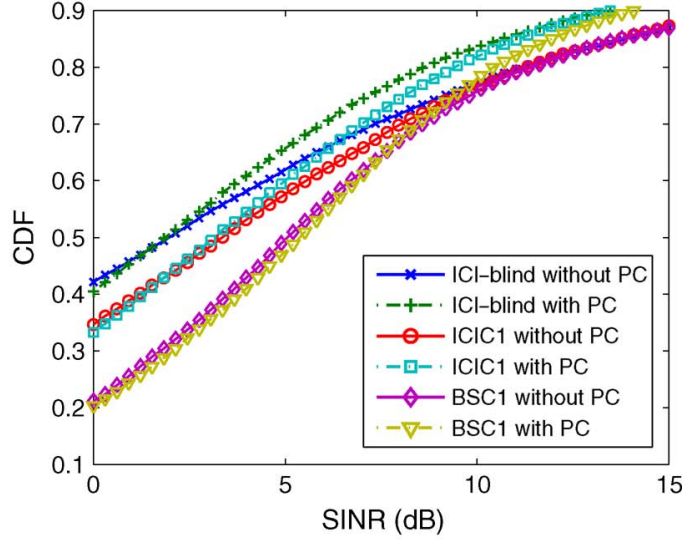

(b)

Fig. 10. SINR distribution for schemes with and without PC (25 MSs per cell). (a) Low-SINR region. (b) High-SINR region.

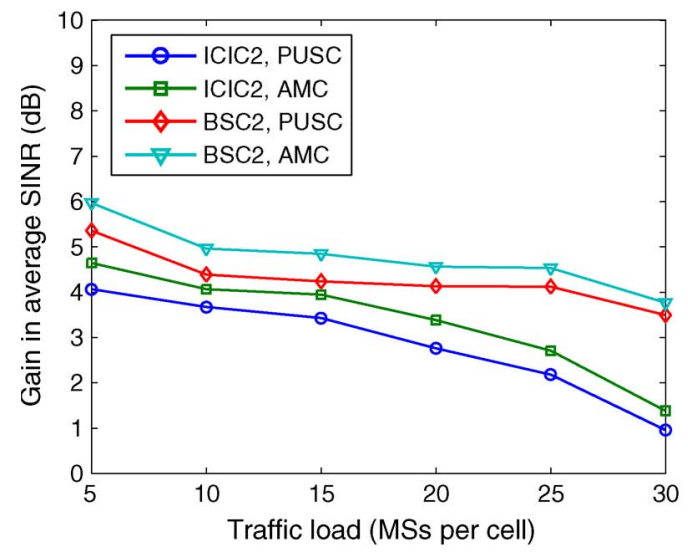

(a)

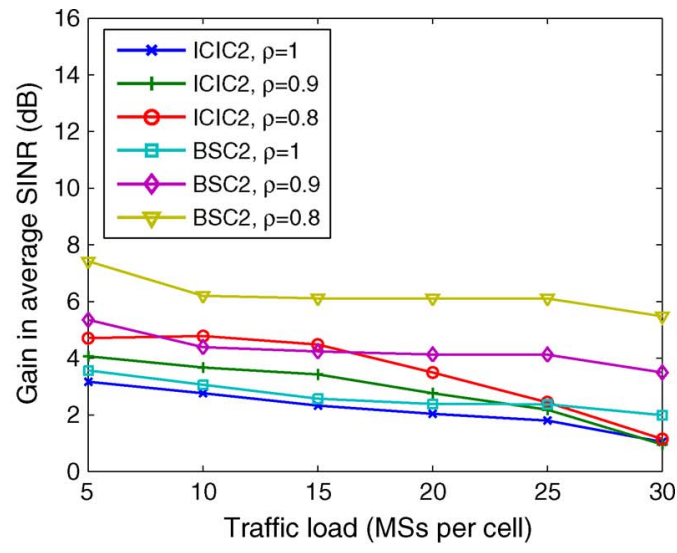

(b)

Fig. 11. Average SINR gains with respect to the ICI-blind scheme under different traffic loads for (a) PUSC and AMC permutation schemes and (b) different intercell distance deployment.

bandwidth allocation in Fig. 1(b) and (c). In each figure, a marker indicates an MS in the corresponding sector (i.e., the $y$-axis) using the corresponding subchannel (i.e., the $x$-axis). Each subfigure shows spectrum allocation for sectors: $A^{1}, A^{4}$, and $A^{5}$ for the top cell index, $B^{1}, B^{2}$, and $B^{3}$ for the middle cell index, and $C^{1}, C^{6}$, and $C^{7}$ for the bottom cell index. The reuse of the same subchannel in physically close areas is viewed as a collision, as shown by connecting lines. A comparison between Fig. 13(a) and (b) readily suggests that our algorithms achieve ICI reduction by lowering the number of collided subchannel assignment. BSC is interesting in that it purposefully allocates the same subchannels to adjacent areas, as depicted in Fig. 1(c). In fact, the "collisions" of subchannels in Fig. 13(c) are for the BSC purpose. Thus, instead of impairing the performance, such "collisions" enhance the overall performance. 


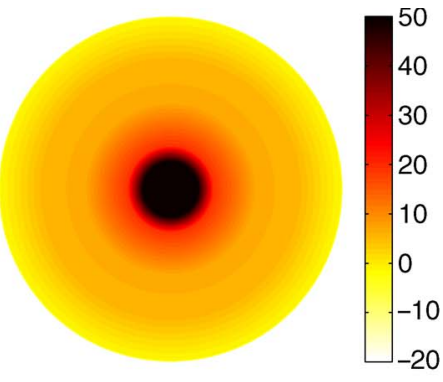

(a)

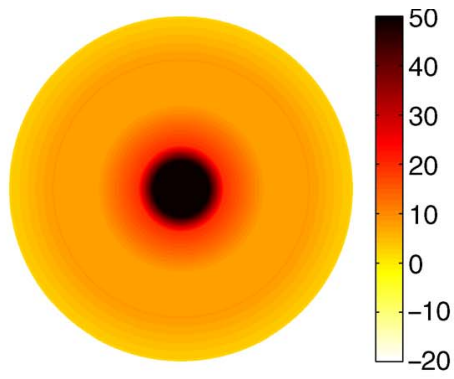

(b)

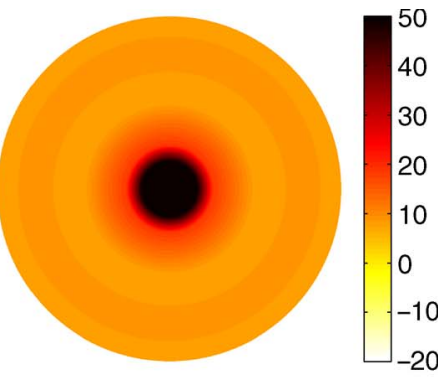

(c)

Fig. 12. Contour plot of SINR (in decibels) in a cell, with 25-MS traffic load per cell. (a) ICI-blind. (b) ICIC1. (c) BSC1.

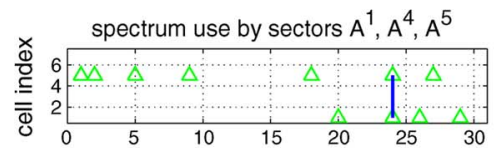

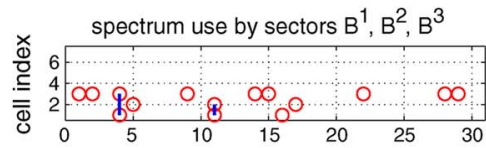

$\times \quad$ spectrum use by sectors $C^{1}, C^{6}, C^{7}$

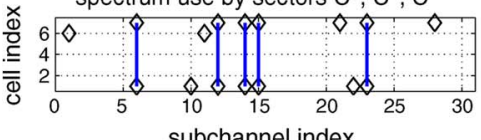

(a)

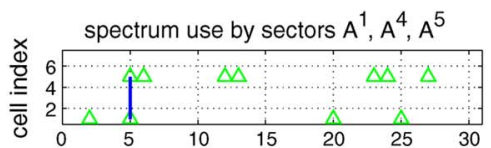

spectrum use by sectors $B^{1}, B^{2}, B^{3}$
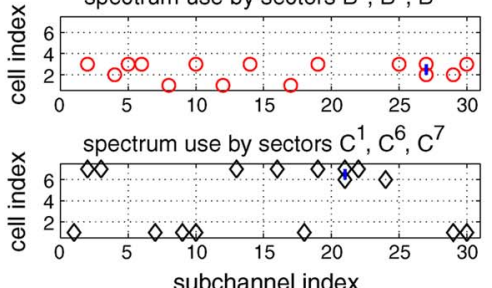

(b)
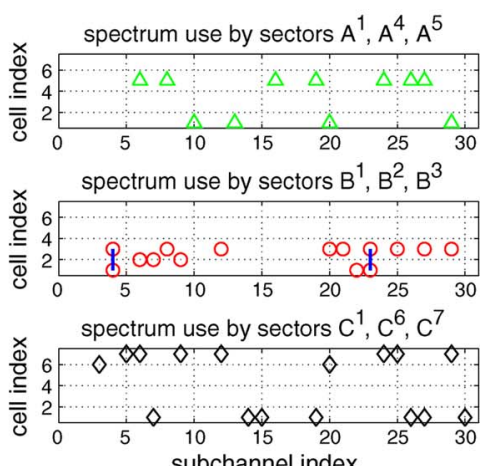

(c)

Fig. 13. Spectrum allocation in ICI-prone areas, where each figure shows spectrum allocation for sectors $A^{1}, A^{4}$, and $A^{5}$ for the top cell index, $B^{1}, B^{2}$, and $B^{3}$ for the middle cell index, and $C^{1}, C^{6}$, and $C^{7}$ for the bottom cell index in Fig. 1(b) and (c). (a) ICI-blind. (b) ICIC1. (c) BSC1.

\section{CONCLUSION}

A downlink multicell OFDMA resource-allocation framework has been proposed in this paper. A two-phase approach with coarse-scale ICI management and fine-scale channelaware allocation has been presented. In particular, the main task of managing the performance-limiting ICI in cellular networks was accomplished by a graphic approach in which state-of-the-art ICI management schemes such as ICIC and BSC can easily be incorporated. A separate handling of interference management and network capacity maximization in the proposed graph framework can deliver a substantial SINR performance improvement, which was confirmed by extensive computer simulation. Due to its practicality and low complexity, the proposed scheme can be used in next-generation cellular systems such as 3GPP LTE and IEEE 802.16m.

\section{REFERENCES}

[1] 3GPP Long-Term Evolution (LTE). [Online]. Available: http://www. 3gpp.org/Highlights/LTE/LTE.htm

[2] IEEE 802.16 Task Group m. [Online]. Available: http://ieee802.org/ $16 / \mathrm{tgm} /$

[3] I. Katzela and M. Naghshineh, "Channel assignment schemes for cellular mobile telecommunication systems: A comprehensive survey," IEEE Pers. Commun., vol. 3, no. 3, pp. 10-31, Jun. 1996.

[4] C. Y. Wong, R. S. Cheng, K. B. Letaief, and R. D. Murch, "Multiuser OFDM with adaptive subcarrier, bit, and power allocation," IEEE J. Sel. Areas Commun., vol. 17, no. 10, pp. 1747-1758, Oct. 1999.

[5] G. Li and H. Liu, "Dynamic resource allocation with finite buffer constraint in broadband OFDMA networks," in Proc. IEEE WCNC, Mar. 2003, pp. 1037-1042.

[6] H. Yin and H. Liu, "An efficient multiuser loading algorithm for OFDM-based broadband wireless systems," in Proc. IEEE GLOBECOM, Nov. 2000, pp. 103-107.
[7] G. Li and H. Liu, "Downlink radio resource allocation for multicell OFDMA system," IEEE Trans. Wireless Commun., vol. 5, no. 12, pp. 3451-3459, Dec. 2006.

[8] S. Hamouda, S. Tabbane, and P. Godlewski, "Improved reuse partitioning and power control for downlink multicell OFDMA systems," in Proc. BWAN, Sep. 2006.

[9] S. Pietrzyk and G. J. Janssen, "Subcarrier allocation and power control for QoS provision in the presence of CCI for the downlink of cellular OFDMA systems," in Proc. IEEE VTC, Apr. 2003, pp. 2221-2225.

[10] S. Pietrzyk and G. J. Janssen, "Radio resource allocation for cellular networks based on OFDMA with QoS guarantees," in Proc. IEEE GLOBECOM, Nov. 2004, pp. 2694-2699.

[11] A. Abrardo, A. Alessio, P. Detti, and M. Moretti, "Centralized radio resource allocation for OFDMA cellular systems," in Proc. IEEE ICC, Jun. 2007, pp. 5738-5743.

[12] G. Fodor, "Performance analysis of a reuse partitioning technique for OFDM based evolved UTRA," in Proc. IEEE IWQoS, Jun. 2006, pp. 112-120.

[13] H. Zhang, N. B. Mehta, A. F. Molisch, J. Zhang, and H. Dai, "On the fundamentally asynchronous nature of interference in cooperative base station systems," in Proc. IEEE ICC, Jun. 2007, pp. 6073-6078.

[14] Feasibility Study for Evolved Universal Terrestrial Radio Access (E-UTRA), 2006.

[15] Evolved UTRA and UTRAN Radio Access Architecture and Interfaces, 2006.

[16] "On Inter-Cell Interference Coordination Schemes Without/With Traffic Load Indication," 3GPP TSG-RAN WG1 Meeting \#50, Oct. 2007.

[17] "Performance Analysis and Simulation Results of Uplink ICIC," 3GPP TSG-RAN WG1 Meeting \#51, Jan. 2008.

[18] IEEE C802.16m-07/244, Collaborative MIMO, Nov. 2007.

[19] "Inter-Cell Interference Handling for E-UTRA," 3GPP TSG-RAN WG1 Meeting \#42, Aug. 2005.

[20] "Interference Mitigation in Evolved UTRA/UTRAN," 3GPP TSG-RAN WG1 Meeting \#42, Aug. 2005.

[21] Y. Chang, Z. Tao, J. Zhang, and C.-C. J. Kuo, "A graph-based approach to multicell OFDMA downlink resource allocation," in Proc. IEEE GLOBECOM, Nov. 2008, pp. 1-6.

[22] Part 16: Air Interface for Fixed and Mobile Broadband Wireless Access Systems, IEEE Std. 802.16e-2005, Feb. 2006. 
[23] M. K. Karakayali, G. J. Foschini, and R. A. Valenzuela, "Network coordination for spectrally efficient communications in cellular systems," IEEE Wireless Commun., vol. 13, no. 4, pp. 56-61, Aug. 2006.

[24] I. Koutsopoulos, T. Ren, and L. Tassiulas, "The impact of space division multiplexing on resource allocation: A unified approach," in Proc. IEEE INFOCOM, Mar. 2003, pp. 533-543.

[25] H. Yin and H. Liu, "Performance of space-division multiple-access (SDMA) with scheduling," IEEE Trans. Wireless Commun., vol. 1, no. 4, pp. 611-618, Oct. 2002.

[26] L. Narayanan, "Channel assignment and graph multicoloring," in Handbook of Wireless Networks and Mobile Computing. Hoboken, NJ: Wiley, 2002, pp. 71-94.

[27] C. McDiarmid and B. Reed, "Channel assignment and weighted coloring," Networks, vol. 36, no. 2, pp. 114-117, Sep. 2000.

[28] F. Y. L. Chin, Y. Zhang, and H. Zhu, "A 1-local 13/9-competitive algorithm for multicoloring hexagonal graphs," in Proc. COCOON, Jul. 2007, pp. 526-536.

[29] S. Sahni and T. Gonzalez, "P-complete approximation problems," J. Assoc. Comput. Mach., vol. 23, no. 3, pp. 555-565, Jul. 1976.

[30] B. Korte and J. Vygen, Combinatorial Optimization: Theory and Algorithms, 3rd ed. New York: Springer-Verlag, 2006.

[31] IEEE 802.16 Broadband Wireless Access Working Group, Draft IEEE 802.16m Evaluation Methodology, 2007.

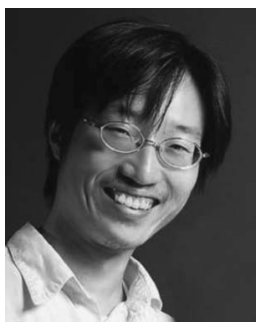

Ronald Y. Chang received the B.S. degree in electrical engineering from the National Tsing Hua University, Hsinchu, Taiwan, in 2000, the M.S. degree in electrical engineering from the National Chiao Tung University, Hsinchu, in 2002, and the Ph.D. degree in electrical engineering from the University of Southern California (USC), Los Angeles, in 2008.

From 2002 to 2003, he was with the Industrial Technology Research Institute, Hsinchu. Since 2004, he has conducted research with USC as well as with the Mitsubishi Electric Research Laboratories, Cambridge, MA, which he visited from January to August 2008. He is currently with the Ming Hsieh Department of Electrical Engineering and the Signal and Image Processing Institute, USC. He has published eight papers in journals and conference proceedings. $\mathrm{He}$ is the holder of five awarded or pending U.S. patents. His research interests include resource allocation and interference management for cellular networks, medium access control protocol design and analysis, quality-of-service provision, and consideration for wireless networks. His research in these areas has established notable academic and industrial values.

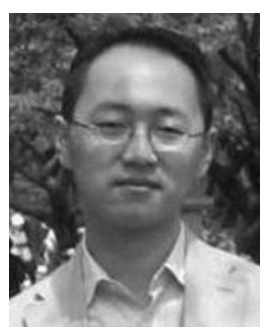

Zhifeng Tao (S'01-M'06) received the B.S. degree from Xi' an Jiaotong University, Xi'an, China, in 2000 and the M.S. and Ph.D. degrees in electrical engineering from the Polytechnic University, Brooklyn, NY, in 2002 and 2006, respectively.

Since September 2006, he has been with the Digital Communications and Networking Group, Mitsubishi Electric Research Laboratories, Cambridge, MA. Since 2004, he has been an active Participant in the IEEE 802.11 standardization and is currently developing the IEEE $802.16 \mathrm{j} / \mathrm{m}$ standard. He has published more than 20 papers and is the author of several book chapters. He is the holder of more than 50 U.S. patents and has made more than 50 technical contributions to IEEE Standards. He is a Coeditor of a special issue of the International Journal of Autonomous and Adaptive Communications Systems. His research interests include wireless communications and networking.

Dr. Tao is a member of the IEEE Communications Society and a Voting Member of IEEE 802.16. He is the Technical Program Committee (TPC) Chair of the Frontier on Communications and Networking Symposium Third IEEE International Conference on Communications and Networking in China (ChinaCom 2008). He was the Publications Chair at the First International Conference on Ambient Media and Systems (AmbiSys 2008) and was a TPC member at several conferences, including the IEEE Global Telecommunications Conference (GLOBECOM), the IEEE International Conference on Communications (ICC), the IEEE International Symposium on Personal, Indoor, and Mobile Radio Communications (PIMRC), and ChinaCom.

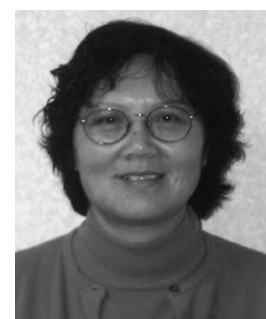

Jinyun Zhang (S'86-M'91-SM'04-F'08) received the B.Sc. degree in radio electronics from Tsinghua University, Beijing, China, in 1970 and the Ph.D. degree in electrical engineering from the University of Ottawa, Ottawa, ON, Canada, in 1991

From 1970 to 1985, she was with Tsinghua University. She then joined Nortel Networks, where she held various management and engineering positions of increasing responsibility in digital signal processing, wireless communication, and optical networks. Since 2001, she has been the Manager of the Digital Communications and Networking Group, Mitsubishi Electric Research Laboratories, Cambridge, MA, where she is currently leading several new broadband wireless communications and networking research projects, including ultrawideband, ZigBee ad hoc networking, multiple-input-multiple-output, broadband multimedia home networking, wireless sensor networks, high-speed wireless local area networks, cooperative communications, WiMAX, and nextgeneration mobile communications systems. She is the author or a coauthor of more than 110 publications and is the holder or a coholder of more than 80 patents and patent applications. She has made numerous contributions to international wireless communications standards.

Dr. Zhang is a member of the IEEE Antennas and Propagation, IEEE Broadcast Technology, IEEE Communications, IEEE Information Theory, IEEE Intelligent Transportation Systems, IEEE Lasers and Electro-Optics, IEEE Signal Processing, and IEEE Vehicular Technology Societies. She is an Associate Editor of the IEEE TRANSACTIONS ON BROADCASTING and was a member of the Technical Program Committee at several IEEE conferences.

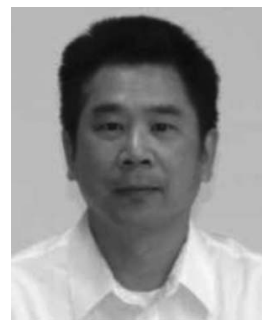

C.-C. Jay Kuo (S'83-M'86-SM'92-F'99) received the B.S. degree in electrical engineering from the $\mathrm{Na}$ tional Taiwan University, Taipei, Taiwan, in 1980 and the M.S. and Ph.D. degrees in electrical engineering from the Massachusetts Institute of Technology, Cambridge, in 1985 and 1987, respectively.

From October 1987 to December 1988, he was a Computational and Applied Mathematics Research Assistant/Professor with the Department of Mathematics, University of California, Los Angeles. Since January 1989 , he has been with the University of Southern California, Los Angeles, where he is currently a Professor of electrical engineering, computer science, and mathematics with the Ming Hsieh Department of Electrical Engineering and the Director of the Signal and Image Processing Institute. He is the Editor-in-Chief of the Journal of Visual Communication and Image Representation and an Editor of the Journal of Information Science and Engineering and the EURASIP Journal of Applied Signal Processing. He is a coauthor of about 150 journal papers, 770 conference proceedings, and nine books. He has guided about 90 students to their $\mathrm{Ph} . \mathrm{D}$. degrees and supervised 20 postdoctoral Research Fellows. His research interests include digital signal and image processing, multimedia compression, and communication and networking technologies.

Dr. Kuo is a Fellow of the International Society for Optical Engineers and a member of the Association for Computing Machinery. From 2003 to 2004, he was on the Editorial Board of the IEEE Signal Processing Magazine. $\mathrm{He}$ was the Associate Editor for the IEEE TRANSACTIONS ON IMAGE PROCESSING during 1995-1998, the IEEE TRANSACTIONS ON CIRCUITS AND SYSTEMS FOR VIDEO TECHNOLOGY during 1995-1997, and the IEEE TRANSACTIONS ON SPEECH AND AUdio Processing during 2001-2003. He received the National Science Foundation Young Investigator Award and the Presidential Faculty Fellow Award in 1992 and 1993, respectively. 